\title{
Thinly-coated hollow core fiber for improved thermal phase-stability performance
}

\author{
Bo Shi ${ }^{1}$, Hesham Sakr ${ }^{1}$, John Hayes ${ }^{1}$, Xuhao Wei $^{1}$, Eric Numkam Fokoua ${ }^{1}$, \\ Meng Ding ${ }^{1}$, Zitong Feng ${ }^{1}$, Giuseppe Marra ${ }^{2}$, Francesco Poletti ${ }^{1}$, David J. \\ RICHARDSON $^{1}$, AND RADAN SLAVÍk ${ }^{1 *}$
}

\author{
${ }^{1}$ Optoelectronics Research Centre, University of Southampton, Southampton, SO17 1BJ, UK \\ ${ }^{2}$ Natinal Physical Laboratory, Hampton Rd., Teddington, TW11 OLW, UK \\ *r.slavik@soton.ac.uk
}

Received XX Month XXXX; revised XX Month, XXXX; accepted XX Month XXXX; posted XX Month XXXX (Doc. ID XXXXX); published XX Month XXXX

The optical phase accumulated when light propagates through an optical fiber changes with temperature. It has been shown by various authors that this thermal phase sensitivity is significantly smaller in Hollow Core Fibers (HCFs) than in Standard Single-Mode Fibers (SSMFs). However, there have been considerable differences in the level of sensitivity reduction claimed, with factors in the range $\times 3$ to $\times 20$ improvement for $\mathrm{HCF}$ relative to SSMF reported. Here, we show experimentally that this large variation is likely attributable to the influence of fiber coating, which is exacerbated in HCFs with a relatively thin silica glass outer wall (e.g., the wall thickness is typically just $20 \mu \mathrm{m}$ in a $125 \mu \mathrm{m}$ diameter HCF). Further, we show that the coating also causes the optical phase stability to suffer from relaxation effects which has not been previously discussed in the HCF literature to the best of our knowledge. As well as demonstrating these relaxation effects experimentally, we also analyze them through numerical simulations. Our results strongly suggest that they originate from the viscoelastic properties of the coating. To minimize the adverse effects of the coating we have fabricated a HCF with a relatively thick wall $(\sim 50 \mu \mathrm{m})$ and a very thin coating $(10 \mu \mathrm{m})$. This resulted in an almost 30 -fold reduction in HCF thermal phase sensitivity relative to SSMF - a significantly lower sensitivity than in previous reports. Moreover, our thinlycoated HCF exhibits no discernable relaxation effects whilst maintaining good mechanical properties.

http://doi.org/XXXXXXXXXXXXX
The optical phase accumulated during the propagation of light through an optical fiber varies due to environmental changes (temperature, vibrations, etc.), which is undesirable for many applications. For example, in interferometers, which lie at the heart of some of the most precise instruments that exist today (e.g., for use in metrology, fiber gyroscopes [1], gravitational wave detectors [2], etc.), this unwanted thermal effect limits the achievable measurement sensitivity and accuracy.

The temperature sensitivity of the accumulated phase of light propagating through a length $\mathrm{L}$ of fiber $\left(\varphi=2 \pi \mathrm{n}_{\mathrm{eff}} \mathrm{L} / \lambda\right)$ can be quantified through:

$$
\mathrm{S}_{\varphi}=\frac{1}{\mathrm{~L}} \frac{\mathrm{d} \varphi}{\mathrm{dT}}=\frac{2 \pi}{\mathrm{L} \lambda} \frac{\mathrm{d}\left(\mathrm{n}_{\mathrm{eff}} \mathrm{L}\right)}{\mathrm{dT}}=\frac{2 \pi}{\lambda}\left(\frac{\mathrm{dn}_{\mathrm{eff}}}{\mathrm{dT}}+\frac{\mathrm{n}_{\mathrm{eff}}}{\mathrm{L}} \frac{\mathrm{dL}}{\mathrm{dT}}\right),
$$

where $n_{\text {eff }}$ is the effective refractive index, $\lambda$ is the wavelength in vacuum, and $T$ is the temperature. Please, note we normalize $S_{\varphi}$ to unit physical fiber length, while in the literature, it is sometimes normalized to unit optical path length (which in silica fibers is about 1.45 times larger, while in hollow core optical fibers, HCFs, it is almost identical to the physical length $L$ ).

In Eq. (1), the first term on the right-hand side represents contributions from changes due to the thermo-optic effect, while the second term relates to the thermally-induced fiber elongation. In standard solid glass-core single-mode silica-based fibers (SSMFs), the first term accounts for $95 \%$ of the contribution to $S_{\varphi}$, which is typically around $48 \mathrm{rad} / \mathrm{m} / \mathrm{K}$ at a wavelength of $1550 \mathrm{~nm}$ $[3,4]$. In HCFs, this term is negligible, leading to the thermal sensitivity of HCF being significantly lower than that of SSMF and typically limited by the second term in Eq. (1) (thermally-induced fiber elongation). Considering the widely-used value of the coefficient of thermal expansion $\left(\alpha_{\text {silica }}\right)$ of fused silica glass at 
room temperature of $0.55 \mathrm{ppm} / \mathrm{K}$ [5], the second term in Eq. (1) should in HCF $\left(n_{\text {eff }} \sim 1\right)$ induce $S_{\varphi}=2.2 \mathrm{rad} / \mathrm{m} / \mathrm{K}$. This is close to the values published for HCFs [6] of $0.52 \mathrm{ppm} / \mathrm{K}$ (corresponding to a $\mathrm{S}_{\varphi}$ of $\left.2.1 \mathrm{rad} / \mathrm{m} / \mathrm{K}\right)$. The coefficient $\alpha_{\text {silica }}$ gets smaller at lower temperatures and crosses zero around $-80^{\circ} \mathrm{C}$ [5], enabling $\mathrm{S}_{\varphi}=0$ (zero phase sensitivity) of HCF to be achieved. This has been confirmed experimentally, with $\mathrm{S}_{\varphi}=0$ being reported at $-71^{\circ} \mathrm{C}$ [7].

The above analysis strictly-speaking applies for uncoated fibers only. The acrylate coatings typically used on optical fibers for protection and to improve their properties have significantly higher coefficients of thermal expansion than fused silica. When a fiber is heated, the coating elongates the fiber beyond what is expected for fused silica. Dangui et al [8] used a simple model to account for this coating contribution. The thermal coefficient of expansion of a single-material coated fiber in this model is:

$$
\alpha_{\text {coated_fib }}=\frac{\alpha_{\text {silica }} E_{\text {silica }} A_{\text {silica }}+\alpha_{\text {coating }} E_{\text {coating }} A_{\text {coating }}}{E_{\text {silica }} A_{\text {silica }}+E_{\text {coating }} A_{\text {coating }}},
$$

where $A$ denotes the cross-sectional area and $E$ the Young's modulus of silica glass and the coating material. Indeed, this model can be straightforwardly extended to more layers [8]. We will consider room temperature $\left(25^{\circ} \mathrm{C}\right)$ and the representative coating parameters given in Ref. [3]: $E_{\text {coating }}=158 \mathrm{MPa}, \alpha_{\text {coating }}=180 \mathrm{ppm} / \mathrm{K}, E_{\text {silica }}=72.5 \mathrm{GPa}$ and $\alpha_{\text {silica }}=0.55 \mathrm{ppm} / \mathrm{K}$. Given $E_{\text {coating }} \ll E_{\text {silica }}$ and considering $A_{\text {coating }} \sim A_{\text {silica }}$, we can neglect the second term in the Eq. (2) denominator, simplifying Eq. (2) to:

$$
\alpha_{\text {coated_fib }} \cong \alpha_{\text {silica }}+\alpha_{\text {coating }} \frac{E_{\text {coating }}}{E_{\text {silica }}} \frac{A_{\text {coating }}}{A_{\text {silica }}} \text {. }
$$

Considering the above numbers, we get:

$$
\alpha_{\text {coated_fib }} \cong 0.55 \mathrm{ppm} / \mathrm{K}+0.39 \mathrm{ppm} / \mathrm{K} \frac{A_{\text {coating }}}{A_{\text {silica }}} .
$$

We see that for a coating $A_{\text {coating }}$ similar or larger than $A_{\text {silica, }}$ the coating may strongly increase the overall fiber expansion. We believe this coating contribution lies behind most of the discrepancies in the $S_{\varphi}$ measured for HCFs in the literature (e.g., $[3,8,9]$ ). Unfortunately, we cannot quantify this, as the data in the literature does not give all the necessary information, e.g., the coating diameter is often not given. However, it seems that the relatively higher values of thermal sensitivity are measured with fibers with relatively small glass fiber cross-sectional area $[8,9]$. By contrast the smaller $\mathrm{S}_{\varphi}$ values reported $[3,4,6,10]$ are mostly on HCFs manufactured at the University of Southampton, where relatively large glass cladding diameters (e.g., 180-260 $\mu \mathrm{m}$ ) are generally used with a corresponding glass cross-sectional area that is about two times larger than for other HCFs, e.g., as measured in [8]. It is worth mentioning that the discrepancy in the reported values is often further exacerbated by different normalization approaches as we mentioned earlier (giving a further discrepancy of about 1.45 times).

In this letter, we firstly manufactured a HCF with a very thin coating $(10 \mu \mathrm{m})$ and a relatively thick glass wall $(50 \mu \mathrm{m})$, which reduces the coating's contribution to $\alpha_{\text {coated_fib }}$ to less than $18 \%$, providing both a practical solution (as the fiber is not as fragile as would be the case for an uncoated fiber [3]), and a low thermal sensitivity. We measured the thermal sensitivity and compared it to that of the standard-thickness coated HCF reported earlier [6]. To further study the coating contribution to $S_{\varphi}$, we also measured a HCF that would be expected to have a significantly larger coating contribution than in our previously studied HCF. This is achieved by using a coating typically employed as a secondary fiber coating in dual-coated fibers which has a significantly larger Young's modulus. We show that besides degrading $\mathrm{S}_{\varphi}$, such a coating also introduces time-dependent creep, which we confirm by comparing measured data with simulations.

The manufactured thinly-coated HCF was of a NANF geometry [11] with a glass silica jacket tube of $185 / 70 \mu \mathrm{m}$ outer/inner diameter, see Fig. 1 . The coating was $10 \mu \mathrm{m}$ thick. Eq. (4) then gives $\alpha_{\text {coated_fib }} \cong 0.65 \mathrm{ppm} / \mathrm{K}$, which is only marginally larger than that expected of the uncoated fiber $(0.55 \mathrm{ppm} / \mathrm{K})$.

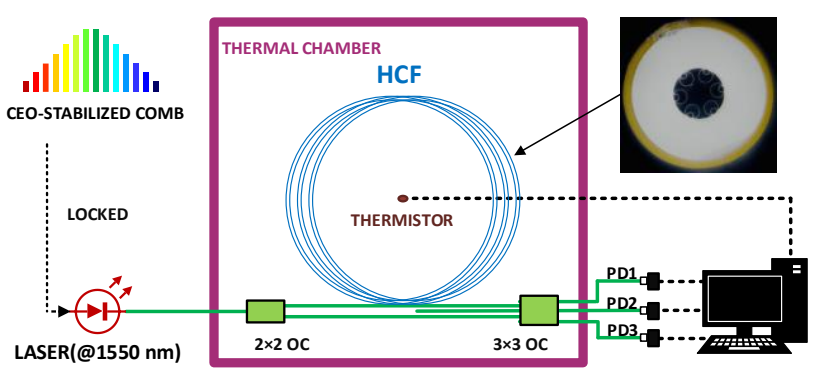

Fig. 1. Measurement set-up used to characterize fiber thermal sensitivity and photograph of the manufactured thinly-coated HCF. CEO-stabilized comb: carrier envelope offsetstabilized optical frequency comb; OC: optical coupler; PD: photodetector.

The second sample we measured was chosen to get an appreciable contribution from the coating. As none of the fibers available to us had a small $A_{\text {silica, }}$, we used a fiber with a relatively large $A_{\text {silica }}$, but dual-coated, as the secondary fiber coating typically has a significantly larger Young's modulus than the single-coating, creating a more prominent effect, as follows from Eq. (3). Neglecting the influence of the soft inner coating and using $E_{\text {out_coating }}=780 \mathrm{MPa}$, Eq. (4) gives $\alpha_{\text {dual_coated_fib }} \cong 0.93 \mathrm{ppm} / \mathrm{K}$, which shows a significant contribution from the coating to the overall fiber elongation ( $\sim 70 \%$ larger than that of the silica glass).

The experimental set-up used to measure the thermal sensitivities of both HCF samples is shown in Fig. 1. We employ a narrow linewidth laser (RIO Orion from NuFern, emitting at $1558 \mathrm{~nm}$ ), which is frequency locked to a carrierenvelope offset (CEO) stabilized optical frequency comb to eliminate laser carrier frequency drift, which ensures the measured phase response is only due to the thermallyinduced phase change in the fibers inside the interferometer. The fibers under test (thinly coated HCF: $24 \mathrm{~m}$; dual-coated HCF: $30.2 \mathrm{~m}$ ) were coiled with a diameter of $14 \mathrm{~cm}$, and spliced into a Mach-Zehnder interferometer (MZI) made of a $2 \times 2$ input coupler and a $3 \times 3$ output coupler, both made of SSMF with tails shorter than $1.5 \mathrm{~m}$. These tails were cut to the same length and placed together to minimize any relative change in optical phase between them. The $3 \times 3$ output 
coupler enables un-ambiguous phase change extraction including its sign [12], which is not possible when a $2 \times 2$ coupler is used. We did not use any polarization control in the interferometer, since a slow drift in polarization that causes change of the interference contrast was accounted for in data processing. We put the interferometer into a thermal chamber and placed a thermistor in the center of the coiled fiber under test.

Fig. 2a shows the measured accumulated phase change in response to temperature change. For the thinly-coated HCF, the phase change follows the temperature change (e.g., when the temperature became stable, the accumulated phase was constant) and the extracted phase sensitivity (calculated from the phase difference at the three temperature points) is shown in Fig. 3. The situation is different for the dual-coated HCF, Fig. $2 b$, where the phase keeps changing even when the temperature is stable. Following a temperature rise (from 20 to $38^{\circ} \mathrm{C}$ and then from 38 to $59^{\circ} \mathrm{C}$ ), the measured phase initially rose, but then continued to reduce for several hours. When decreasing the temperature, we observe the same effect but with an opposite sign. The drift of phase at constant temperature eventually stopped when the temperature was kept constant. In Fig. $2 \mathrm{~b}$, we see this for example at the temperature of $59^{\circ} \mathrm{C}$ (time of $12-22$ hours) and at $20^{\circ} \mathrm{C}$ (time of 36-60 hours). It looks as if the coating was relaxing over time, reducing its effect on the overall fiber length change. We will discuss this effect in detail later. Besides this timedependent behavior, the overall thermal sensitivity of the dual-coated HCF is larger than for the thinly-coated HCF, as shown in Fig. 3 in which the phase measured at five stable temperature points in Fig. $2 \mathrm{~b}$ is used for the calculation. The error bars correspond to the difference between the values measured when increasing and decreasing the temperature. For comparison, we also plot in Fig. 3 the thermal sensitivity results measured on our typical single-coated HCF (data adopted from [6]).

We see that the thermal sensitivities of the two singlecoated HCFs differ by about $20 \%$, with the thinly-coated HCF achieving a lower sensitivity result than expected from the uncoated HCF (2.2 $\mathrm{rad} / \mathrm{m} / \mathrm{K}$, as discussed earlier). Inserting the thinly-coated HCF thermal sensitivity and the abovementioned coating parameters and fiber dimensions into Eq. (4) gives the silica coefficient of thermal expansion to be 0.3 $\mathrm{ppm} / \mathrm{K}$ (at $25^{\circ} \mathrm{C}$ ). This value is significantly lower than the widely-used value of $0.55 \mathrm{ppm} / \mathrm{K}$ [5]. Even when neglecting the coating influence in Eq. (4), the value is $0.39 \mathrm{ppm} / \mathrm{K}$, which is still significantly lower than expected. This is not in contradiction with values reported in the literature, where fused silica glass has been demonstrated to have a thermal expansion coefficient at room temperature spanning a relatively large range from $0.3 \mathrm{ppm} / \mathrm{K}$ to $0.55 \mathrm{ppm} / \mathrm{K}$ $[13,14,15]$. This result is also consistent with our previous measurements [6] shown in Fig. 3, in which we measured a value of $0.47 \mathrm{ppm} / \mathrm{K}$ (already below that of $0.55 \mathrm{ppm} / \mathrm{K}$ ) even for a single-coated HCF with standard coating thickness. This result is also consistent with our measurement on dualcoated HCF, where using $0.3 \mathrm{ppm} / \mathrm{K}$ in Eq. 4 gives $\alpha_{\text {dual_coated_fib }}=0.68 \mathrm{ppm} / \mathrm{K}$, which corresponds to the measured value $(0.66 \mathrm{ppm} / \mathrm{K}$, calculated from data in Fig. 3). We conclude that the silica coefficient of thermal expansion for optical fibers (and in particular HCF) can be as low as 0.30 $\mathrm{ppm} / \mathrm{K}$.
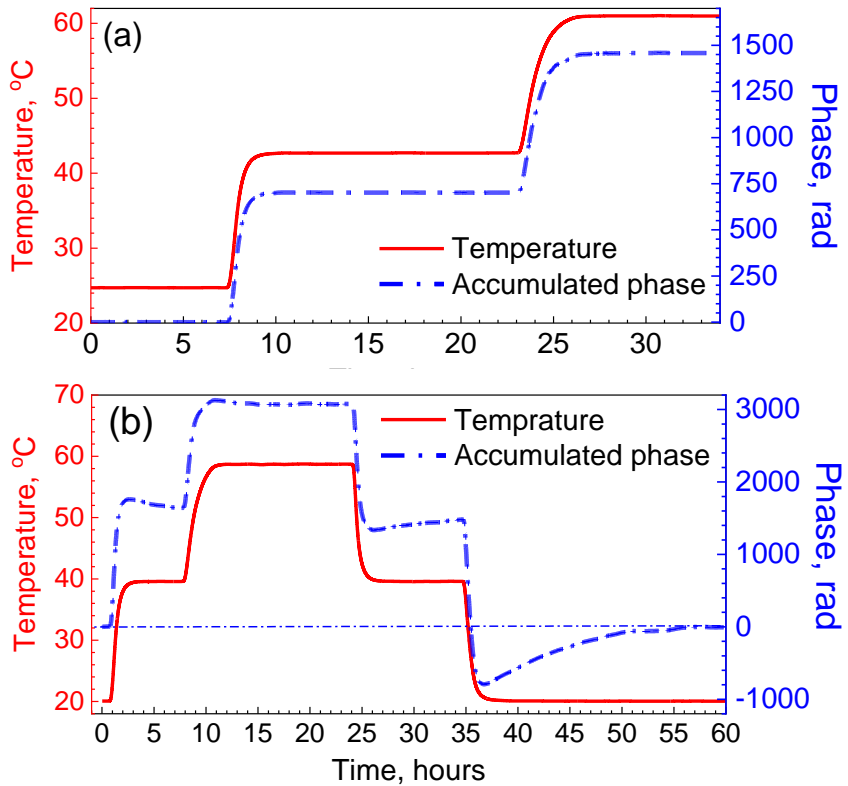

Fig. 2. Temperature inside the thermal chamber and accumulated phase measured for the 24-m long thinly-coated HCF (a) and 30-m long dualcoated HCF (b).

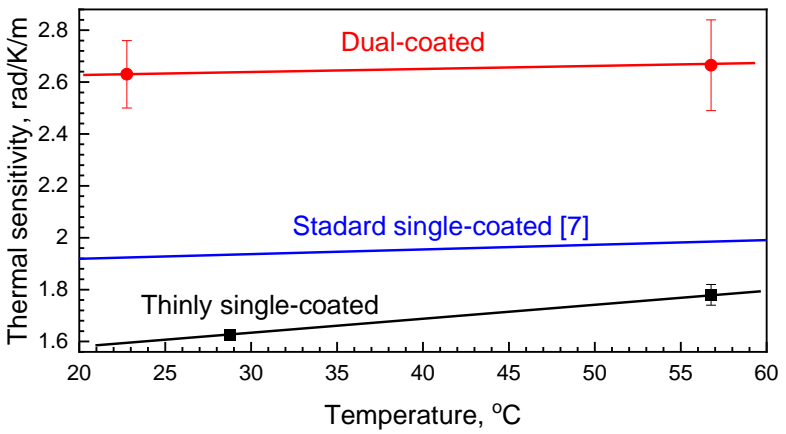

Fig. 3. Phase thermal sensitivity of three measured HCFs (thinly-coated, standard single-coated and dual-coated) calculated from data shown in Fig. 2 (thinly-coated and dual-coated) and from the data in [6] (standard singlecoated).

In Fig. $2 b$ we show that besides a higher value of thermal phase sensitivity, the HCF coating is also responsible for the phase relaxation effect, where the light phase keeps changing many hours after the temperature has been stabilized. To understand better this effect, we modelled the thermal behavior of the dual-coated HCF sample in COMSOL Multiphysics. The diameter of the coating and silica cladding of the modeled fiber were set to be the same as the manufactured sample, while the contribution from the inner tubes (microstructure) to the thermal sensitivity was neglected. In COMSOL, two independent physics interfaces (Solid mechanics and Heat transfer in solids) were employed and coupled together to simulate the thermal elongation of 
an optical fiber, including both heat transfer and the mechanical properties. In particular, we modelled the coating layers as viscoelastic materials, which are characterized (besides the elastic properties) by a relaxation time that describes the relaxation of thermal stress [16]. In the simulations, we considered the temperature observed in the experiment (shown in Fig. 2b). We fitted all the parameters from their initial values obtained in the literature and relevant datasheets, in particular, Young's modulus, thermal expansion coefficients, and relaxation time. Fig. 4 compares the measured (shown in Fig. 2b) and fitted phase changes in response to temperature change. We were able to obtain an excellent fit, especially in term of the relaxation caused by the coating viscoelastic properties. It is worth mentioning that we were not able to obtain a good fit with $\boldsymbol{\alpha}_{\text {silica }}=0.55$ $\mathrm{ppm} / \mathrm{K}$, especially in terms of the relaxation. However, using $\boldsymbol{\alpha}_{\text {silica }}=0.3 \mathrm{ppm} / \mathrm{K}$ as discussed above allowed us to achieve a good fit, as shown in Fig. 4. The fitted time constant of the coating varies between $60000 \mathrm{~s}$ at $25^{\circ} \mathrm{C}$ and $6000 \mathrm{~s}$ at $60^{\circ} \mathrm{C}$. We consider this reasonable, as the other coating parameters (Young's modulus in particular) also change significantly with temperature. To avoid this thermal relaxation induced phase shift, we suggest using a thick HCF silica cladding jacket in conjunction with a thin coating. Such a design also reduces the thermal phase sensitivity of the HCF, as we show here experimentally and as was previously demonstrated numerically [8]. Thick HCF silica jacket should also have additional benefit of reducing the acoustic sensitivity of HCF [17]. Other alternatives to reduce the thermal relaxation effect are to use different coating materials that do not show as strong viscoelastic behavior such as acrylates, e.g. possibly polyimide.

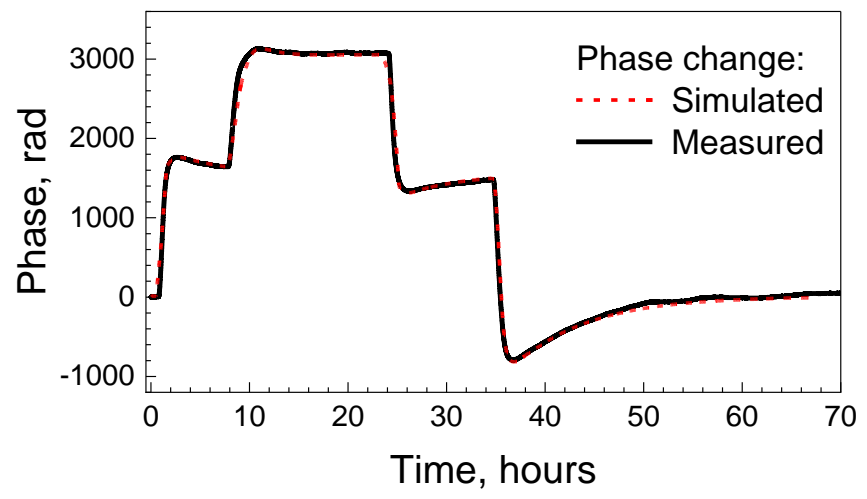

Fig. 4.Comparison of measured (shown in Fig. 2) and simulated thermal sensitivity of dual-coated HCF considering viscoelastic behavior of the coating.

In conclusions, we have shown experimentally that coatings can play a significant role in determining the thermal sensitivity of HCF. We believe this is behind most of the discrepancies in the literature in terms of the thermal sensitivity measured for various HCFs. To minimize its unwanted effect, we fabricated a relatively thick $(185 \mu \mathrm{m}$ diameter) HCF with only $10 \mu \mathrm{m}$ thin acrylate coating. The thin coating did not have any adverse effect on the mechanical strength of the fiber which was kept coiled at 14 $\mathrm{cm}$ diameter over three months. Its thermal sensitivity was lower than expected and we believe this is because the thermal expansion coefficient of silica glass from which the HCF is made is lower than the value often used in the literature. This makes HCF even less thermally sensitive (1.25 times) than the best values previously reported at room temperature [3], making it almost 30 times less sensitive than SSMF (48 rad/m/K, as calculated from the data in [18]).

Finally, we showed that besides compromising the phase sensitivity coefficient, the coating can also cause a drift in the optical phase even when kept at constant temperature for a period and we show this is due to the coating's viscoelastic properties. Besides HCFs, this effect is expected to play a role also in SSMF-based systems, e.g., in delayed-line interferometers uses for laser stabilization [19].

Funding. EPSRC Airguide (EP/P030181/1), RAEng Fellowship (R. Slavík, E. Numkam Fokoua). ERC Lightpipe (F. Poletti). China Scholarship Council (CSC).

Disclosures. The authors declare no conflicts of interest.

Acknowledgment. The data for this work is accessible through the University of Southampton Institutional Research Repository (DOI: 10.5258/SOTON/D1964).

\section{References}

1. V.M.N. Passaro, A. Curccovillo, L. Vaiani, M. De Carlo, and C.E. Capanella, Sensors. 17, 2284 (2017).

2. B. P. Abbott, R. Abbott, T. D. Abbott, M. R. Abernathy, F. Acernese, K. Ackley, C. Adams, T. Adams, P. Addesso, and R. X. Adhikari, Phys. Rev. Lett. 116, 61102 (2016).

3. W. Zhu, E. R. N. Fokoua, A. A. Taranta, Y. Chen, T. Bradley, M. N. Petrovich, F. Poletti, M. Zhao, D. J. Richardson, and R. Slavík, J. Lightwave Technol. 38, 2477 (2019).

4. E. Numkam Fokoua, M. N. Petrovich, T. Bradley, F. Poletti, D. J. Richardson and R. Slavík, Optica. 4, 659 (2017).

5. D. E. Gray, ed., American Institute of Physics Handbook, (McGraw-Hill, 1972).

6. R. Slavík, E. N. Fokoua, M. Bukshtab, Y. Chen, T. Bradley, S. R. Sandoghchi, M. Petrovich, F. Poletti, and D. Richardson, Opt. Lett. 44, 4367 (2019).

7. W. Zhu, E. N. Fokoua, Y. Chen, T. Bradley, M. Petrovich, F. Poletti, M. Zhao, D. Richardson, and R. Slavík, Opt. Lett. 44, 2768 (2019).

8. V. Dangui, H. K. Kim, M. J. Digonnet, and G. S. Kino, Opt. Express 13, 6669 (2005)

9. S. Meiselman, G. A. Cranch, Opt. Express 25, 27581 (2017).

10. F. Azendorf, B. Schmauss, B. Shi, E. N. Fokoua, R. Slavík, and M. Eiselt, in Optical Fiber Communication Conference(OFC). (Optical Society of America, 2021), paper F4C.6.

11. G. T. Jasion, T. D. Bradley, K. Harrington, H. Sakr, Y. Chen, E. N. Fokoua, I. A. Davidson, A. Taranta, J. R. Hayes, and D. J. Richardson, in Optical Fiber Communication Conference(OFC). (Optical Society of America, 2020), paper Th4B. 4.

12. K. Koo, A. Tveten, and A. Dandridge, Appl. Phys. Lett. 41, 616 (1982)

13. G K White, J. Phys. D: Appl. Phys. 6 ,2070 (1973)

14. X. Li, S. Lin, J. Liang, Y. Zhang, H. Oigawa, and T. Ueda, IEEE Photonics .J. 4, 155 (2011).

15. B. Deng, Y. Shi, and F. Yuan, Mater. 12, 100752 (2020).

16. S. Shiue, Y. Tu, J. Appl. Phys. 86, 4085(1999).

17. M. Pang, W. Jin, Opt. Express 17, 11088(2009).

18. R. Slavík, G. Marra, E. N. Fokoua, N. Baddela, N. V. Wheeler, M. Petrovich, F. Poletti, and D. J. Richardson, Sci. Rep. 5, 15447 (2015).

19. F. Kéfélian, H. Jiang, P. Lemonde, and G. Santarelli, Opt. Lett 34, 914(2009). 


\section{References: full}

1. V.M.N. Passaro, A. Curccovillo, L. Vaiani, M. De Carlo, and C.E. Capanella, "Gyroscope tecnology and applications: a review in the industrial perspective," Sensors 17, 2284 (2017).

2. B. P. Abbott, R. Abbott, T. D. Abbott, M. R. Abernathy, F. Acernese, K. Ackley, C. Adams, T. Adams, P. Addesso, and R. X. Adhikari, "Observation of gravitational waves from a binary black hole merger," Phys. Rev. Lett. 116, 61102 (2016).

3. W. Zhu, E. R. N. Fokoua, A. A. Taranta, Y. Chen, T. Bradley, M. N. Petrovich, F. Poletti, M. Zhao, D. J. Richardson, and R. Slavík, "The thermal phase sensitivity of both coated and uncoated standard and hollw core fibers down to cryogenic temperatures," J. Lightwave Technol. 38, 2477-2484 (2019).

4. D. E. Gray, ed., American Institute of Physics Handbook (McGraw-Hill, 1972).

5. E. Numkam Fokoua, M. N. Petrovich, T. Bradley, F. Poletti, D. J. Richardson and R. Slavík, "How to make the propagation time throuth an optical fiber fully insensitive to temperature variations," Optica 4, 659-668 (2017).

6. R. Slavík, E. N. Fokoua, M. Bukshtab, Y. Chen, T. Bradley, S. R. Sandoghchi, M. Petrovich, F. Poletti, and D. Richardson, "Demonstration of opposing thermal sensitivities in hollw-core fibers with open and sealed ends," Opt. Lett. 44, 4367-4370 (2019).

7. W. Zhu, E. N. Fokoua, Y. Chen, T. Bradley, M. Petrovich, F. Poletti, M. Zhao, D. Richardson, and R. Slavík, "Temperature insensitive fiber interferometry," Opt. Lett. 44, 2768-2770 (2019).

8. V. Dangui, H. K. Kim, M. J. Digonnet, and G. S. Kino, "Phase sensitivity to temperature of the fundamental mode in air-guiding photonic-bandgap fibers," Opt. Express 13, 6669-6684 (2005).

9. S. Meiselman, G. A. Cranch, "Optical phase response to temperature in a hollow-core photonic crystal fiber," Opt. Express 25, 27581-27594 (2017).

10. F. Azendorf, B. Schmauss, B. Shi, E. N. Fokoua, R. Slavík, and M. Eiselt, "Hollow-core fiber characterization with correlation-optical time domain reflectometry," in Optical Fiber Communication Conference(OFC). (Optical Society of America, 2021), paper F4C.6.

11. G. T. Jasion, T. D. Bradley, K. Harrington, H. Sakr, Y. Chen, E. N. Fokoua, I. A. Davidson, A. Taranta, J. R. Hayes, and D. J. Richardson, "Hollow core NANF with $0.28 \mathrm{~dB} / \mathrm{km}$ attenuation in the $\mathrm{C}$ and L bands," in Optical Fiber Communication Conference Postdeadline Papers (Optical Society of America, 2020), paper Th4B. 4

12. K. Koo, A. Tveten, and A. Dandridge, "Passive stabilization scheme for fiber interfeometers using $(3 \times 3)$ fiber directional couplers," Appl. Phys. Lett. 41 $616-618(1982)$.

13. G K White, "Thermla expansion of reference materials: copper, silica and silicon," J. Phys. D: Appl. Phys. 6 ,2070-2078 (1973)

14. X. Li, S. Lin, J. Liang, Y. Zhang, H. Oigawa, and T. Ueda, "Fiber-optic temeprature sensor based on difference of thermal expansion coefficient between fused silica and metallic materials," EEE Photonics .J. 4, 155-162 (2011).

15. B. Deng, Y. Shi, and F. Yuan, "Inverstigation on the structural origin of low thermal expansion coefficient of fused silica," Mater. 12, 100752 (2020).

16. S. Shiue, Y. Tu, "Relaxation of thermla stresses in double-coated optical fibers, " J. Appl. Phys. 86, 4085-4089(1999).

17. M. Pang, W. Jin, "Detection of acoustic pressure with hollow-core photonic bandgap fiber," Opt. Express 17, 11088(2009).

18. R. Slavík, G. Marra, E. N. Fokoua, N. Baddela, N. V. Wheeler, M. Petrovich, F. Poletti, and D. J. Richardson, "Ultrlow thermla sensitivity of phase and propagation delay in hollow-core fibres," Sci. Rep. 5, 15447 (2015).

19. F. Kéfélian, H. Jiang, P. Lemonde, and G. Santarelli, "Ultralow-frequencynoise stabilization of a laser by locking to an optical fiber-delay line", Opt. Lett 34, 914-916 (2009). 